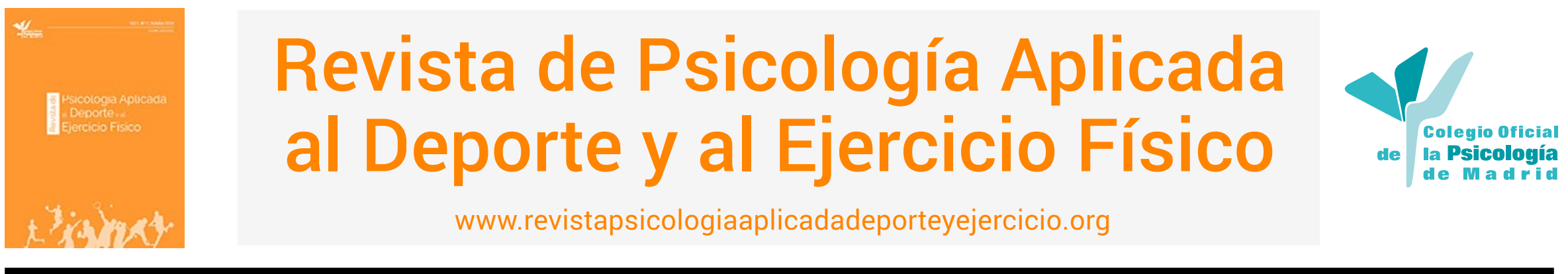

\title{
Análisis de la carrera dual en deportistas peruanos de alto rendimiento
}

\author{
Jorge Moreno-Peña \\ Universidad de Lima, Perú
}

\section{Saúl Chacaliaza Briones}

Universidad Nacional Mayor de San Marcos, Perú

\section{Alex Robles Gutierrez \\ Universidad Nacional Mayor de San Marcos, Perú}

RESUMEN: El objetivo del presente estudio es hacer un análisis descriptivo de la carrera dual (compaginar estudios y deporte) en deportistas peruanos que compiten a nivel internacional. Mediante una encuesta que recogía información sobre datos personales, entorno académico y trayectoria deportiva se obtuvieron los siguientes resultados: las principales dificultades para llevar a cabo una carrera dual (CD) son el factor tiempo y el económico. La mayoría de deportistas no recibieron ningún beneficio económico por parte de su centro de estudio, ni contaron con un tutor deportivo, así mismo, un gran número de deportistas perciben la carrera dual como un proceso difícil de llevar. En cuanto al entorno familiar, los deportistas consideran a la familia como el principal agente social más importante para el desarrollo de la CD. Finalmente, en lo referente a los datos obtenidos sobre las expectativas una vez culminados los estudios se sitúa mayoritariamente en poder trabajar en lo estudiado. Consideramos tener en cuenta los datos obtenidos para el desarrollo de programas con miras a llevar a cabo una carrera dual exitosa.

PALABRAS CLAVES: Deportistas, carrera dual, práctica deportiva, estudiar, trabajar.

\section{Dual career analysis in high-performance Peruvian athletes}

ABSTRACT: A descriptive analysis is made of the dual race (combining studies and sport) in Peruvian athletes who compete at an international level. The following results were obtained through a survey that collected personal data and information on the academic environment and sports career: the main difficulties in carrying out a dual career (DC) were time and economic issues of the students; most of the athletes did not receive any economic benefit from their study center, nor did they have a sports tutor; and a large number of athletes perceived DC as a difficult process to carry out. Regarding the family environment, the athletes considered the family as the most important social agent for the development of DC. Finally, regarding the data obtained on the expectations once the studies were completed, the main focus was on being able to work in what has been studied. In our opinion, due consideration is required of the data obtained for the development of programs, with a view to carrying out a successful dual career.

KEYWORDS: Athletes, dual career, sports practice, study, work.

\footnotetext{
Jorge Moreno-Peña es psicólogo. Profesor de la Facultad de Psicología de la Universidad de Lima, Perú.

Saúl Chacaliaza Briones es bachiller en psicología.

Alex Robles Gutierrez es bachiller en psicología.

La correspondencia sobre este artículo debe enviarse a: Jorge David Moreno-Peña. Facultad de psicología. Av. Javier Prado Este 4600, Santiago de Surco 15023. Lima.Perú. Tel. (+51)950671813. E-mail:dmoreno@ulima.edu.pe
} 


\section{Análise de carreira dupla em atletas peruanos de alta competição}

RESUMO: O objetivo deste estudo é fazer uma análise descritiva da carreira dupla (combinando estudos e desporto) em atletas peruanos que competem internacionalmente. Através de um inquérito que recolheu informações sobre dados pessoais, ambiente académico e trajetória desportiva, obtiveram-se os seguintes resultados: as principais dificuldades na prossecução de uma carreira dupla (CD) são o fator tempo e o fator económico. A maioria dos atletas não recebeu nenhum benefício económico de seu centro de estudo, nem teve um tutor desportivo, além disso, um grande número de atletas encara a carreira dupla como um processo difícil de realizar. Quanto ao ambiente familiar, os atletas consideram a família como o principal agente social mais importante para o desenvolvimento da CD. Finalmente, em relação aos dados obtidos sobre as expectativas após a conclusão dos estudos, cinge-se maioritariamente no facto de poder trabalhar no que foi estudado. Consideramos ter em conta os dados obtidos para o desenvolvimento de programas com vista a prosseguir uma carreira dupla bem-sucedida.

PALAVRAS-CHAVE: Atletas, carreira dupla, prática desportiva, estudo, trabalho.

Artículo recibido: 23/04/2021 | Artículo aceptado: 31/05/2021

Durante los últimos años, los casos de deportistas que alternan entre la carrera deportiva y la carrera académica han venido en aumento, lo cual ha propiciado el auge de numerosas investigaciones al respecto (Álvarez et al., 2014). En el contexto peruano, la alternancia entre carrera deportiva y carrera académica se ha incrementado, por lo que surge la necesidad de estudiar la realidad y los factores que determinan este proceso dentro de la trayectoria de la carrera deportiva y que a su vez nos permitan el abordaje psicológico de una manera adecuada, ya que, de no ser así, puede traer como consecuencia el desequilibrio del desarrollo profesional a futuro (López de Subijana et al., 2015).

La carrera dual, definida como "la combinación del deporte y los estudios que ayuda a los deportistas a lograr sus objetivos académicos y deportivos, vivir una vida privada satisfactoria, manteniendo la salud y el bienestar" (Stambulova et al., 2015, p.24) cumple un papel fundamental en la carrera deportiva. No obstante, una de las dificultades que surgen es comprender la carrera dual. Por ello es conveniente identificar la trayectoria deportiva que desarrolla el deportista a partir de los 3 modelos que plantean Pallarés et al. (2011) donde el modelo lineal es referido a una dedicación exclusiva al deporte sin ninguna formación en otro ámbito, en cuanto al modelo convergente se abarcan estudios alternativos pero aún tiene más relevancia el deporte y respecto al modelo paralelo supone una dedicación distribuida de forma equitativa tanto de la carrera deportiva como de la académica. Asimismo, conocer el modelo holístico de Wylleman (2019) permitirá observar las diferentes circunstancias que el deportista afronta desde una perspectiva global, es decir, integra el desarrollo del deportista de alto nivel con otros contextos que va experimentando a lo largo de su vida como la psicológica, psicosocial, académica / vocacional, económica y legal.
Por otro lado, diversos estudios confirman la importancia del apoyo que recibe el deportista de su entorno para lograr un mejor desarrollo de la carrera dual, tal como lo indica el estudio de Moreno et al. (2018) donde se destaca el papel del apoyo familiar para que el deportista pueda afrontar las demandas académicas y deportivas. Asimismo, Tessitore et al. (2020) resaltan el protagonismo de los padres de los hijos deportistas para un mejor afrontamiento en la carrera dual. Por su parte, Moreno et al. (2020) exploraron la relación entre la formación académica de los padres con el desarrollo de la carrera dual de los deportistas de élite. También podemos encontrar estudios que contribuyeron al mejoramiento de la carrera dual a través de programas como el de Jordana et al. (2019) que describieron la implementación de un Programa de Asistencia de Carrera Dual (PACD) así como otro estudio donde analizaron si un Programa de Asistencia a la Carrera (PROAD) facilita el desarrollo de la carrera dual, ya que, compaginar deportes y estudios es una tarea difícil para la mayoría de deportistas (López de Subijana et al., 2015). Por otro lado, se estudió el éxito académico de un modelo universitario de carrera dual en deportistas-estudiantes en general, teniendo en cuenta el nivel de estudios y el tipo de deporte. (Sánchez-Pato et al., 2018).

Como se puede observar, de acuerdo con los hallazgos expuestos anteriormente, estudiar la carrera dual no solamente implica explorar las variantes internas que ocurren en el deportista, sino también las condiciones externas y extradeportivas que acontecen en su vida cotidiana. Además, es relevante considerar que compaginar deportes con estudios trae diversos beneficios y su promoción permitirá obtener las herramientas necesarias a los deportistas que afronten las etapas de la carrera deportiva, así como a aquellos que no logren la transición al alto rendimiento proporcionándoles alternativas para adaptarse a 
las demandas del mundo externo al deporte (Torregrosa et al., 2016)

Por otro lado, situándonos en el contexto peruano, no existen estudios previos que faciliten la comprensión de la carrera dual. Es por esta razón que el objetivo del presente estudio es describir cómo se desarrolla la carrera dual en deportistas peruanos de alto rendimiento y generar un punto de partida para el desarrollo de diferentes programas de asesoramiento e intervención que faciliten la promoción de la carrera dual desde una perspectiva que considere el nivel cultural general, el cultural deportivo y el entorno del deportista, así como realzar la importancia del papel del psicólogo del deporte como agente activo en el seguimiento del deportista durante su carrera deportiva (Torregrosa et al., 2016).

\section{Método}

\section{Participantes}

En el presente estudio participaron 179 deportistas, de los cuales 101 fueron mujeres (56.42\%) y 78 fueron hombres (43.58\%), con una edad comprendida entre los 19 y 37 años $(M=24.99, D . T= \pm 4.04)$. La muestra estaba conformada por deportistas de alto rendimiento que pertenecen a diferentes federaciones deportivas del Perú, entrenan 6 días a la semana con una media de 4 horas diarias y que, además, han tenido representación internacional en diferentes eventos a nivel sudamericano, panamericano y mundial. El grupo de deportistas estaba agrupado de la siguiente manera: 41 deportistas (23\%) pertenecían a la modalidad de combate (esgrima, karate, lucha y TKD); 85 deportistas (48\%) conformaban los deportes colectivos y de balón (béisbol, bowling, fútbol, balonmano, voleibol, hockey, rugby, softball y waterpolo); en cuanto a los deportes de tiempo y precisión (atletismo, ciclismo, natación, patinaje, tiro deportivo y tiro con arco) fueron evaluados 27 deportistas (15\%), mientras que 15 deportistas (8\%), eran de deportes de raqueta (bádminton, pelota vasca y tenis de mesa) ) y finalmente, 11 deportistas (6\%) pertenecían a los deportes de apreciación (ecuestre, gimnasia artística, natación artística y surf).

\section{Instrumento}

Para llevar a cabo la recogida de datos, se utilizó una encuesta de 27 preguntas elaboradas en base al Cues- tionario de Percepción de los estudiantes-deportistas de alto nivel sobre la carrera dual (Sánchez-Pato et al., 2016) y que pasaron un proceso de validación de jueces para evaluar su coherencia, claridad, relevancia y suficiencia. Éstas eran preguntas de elección múltiple y estaban agrupadas en base a tres secciones: datos personales, entorno académico y carrera dual. Así mismo, se preparó un formato físico y uno virtual para facilitar el llenado de la misma.

\section{Procedimiento}

La recogida de datos se llevó a cabo mediante dos estrategias y fue realizada por licenciados en psicología que cuentan con la especialización y experiencia en el campo de la psicología deportiva. Por un lado, se contactó directamente con los deportistas para visitarlos al lugar de entrenamiento y explicarles el objetivo del estudio. Una vez hecho esto, procedieron a resolver el cuestionario en formato papel.

Por otro lado, en el caso de los deportistas que estaban fuera del país, se les contactó por medio de una videollamada para explicarles el objetivo del estudio, y se realizó el envío del cuestionario online. En ambos casos se aseguró el consentimiento del deportista, como también el anonimato y la confidencialidad de sus respuestas.

\section{Análisis de datos}

Se utilizó el programa IBM SPSS v26 para el procesamiento de los datos. Se calculó la frecuencia (f), porcentaje $(\%)$ y media $(M)$ para realizar el análisis descriptivo de las características de la carrera dual. Asimismo, se emplearon tablas, gráficos de barras y circulares como parte de la presentación de los resultados.

\section{Diseño}

El presente estudio es de enfoque cuantitativo y de diseño transversal descriptivo, ya que se ha recolectado la información en un momento único para conocer los valores en que se manifiestan las variables estudiadas (Gómez, 2015). 


\section{Resultados}

A partir de la información obtenida, los resultados se han agrupado en dos campos específicos. El primero hace referencia a la carrera académica y el segundo a la carrera dual, que involucra compaginar la carrera deportiva y académica.

\section{Datos de carrera académica}

En relación al área académica, los datos considerados han sido agrupados de la siguiente manera: motivos para seguir estudiando, las expectativas al finalizar los estudios, las características de la formación superior y el apoyo económico que reciben los deportistas por parte de la Universidad o Centro de Estudios. En primer lugar, se muestran los resultados en base a la pregunta: ¿Por qué motivos elegiste continuar estudiando? (Figura 1), siendo las principales respuestas: Trabajar en el futuro $(68 \%, n=122)$ y Disfrutar del estudio ( $52 \%, n=93)$. Por otro lado, con puntuaciones muy bajas (menos del 10\%), se ubicaron: Presión familiar (9\%), Interacción social (7\%) y Otros (8\%).

Figura 1. Motivos por los que se realiza una carrera académica

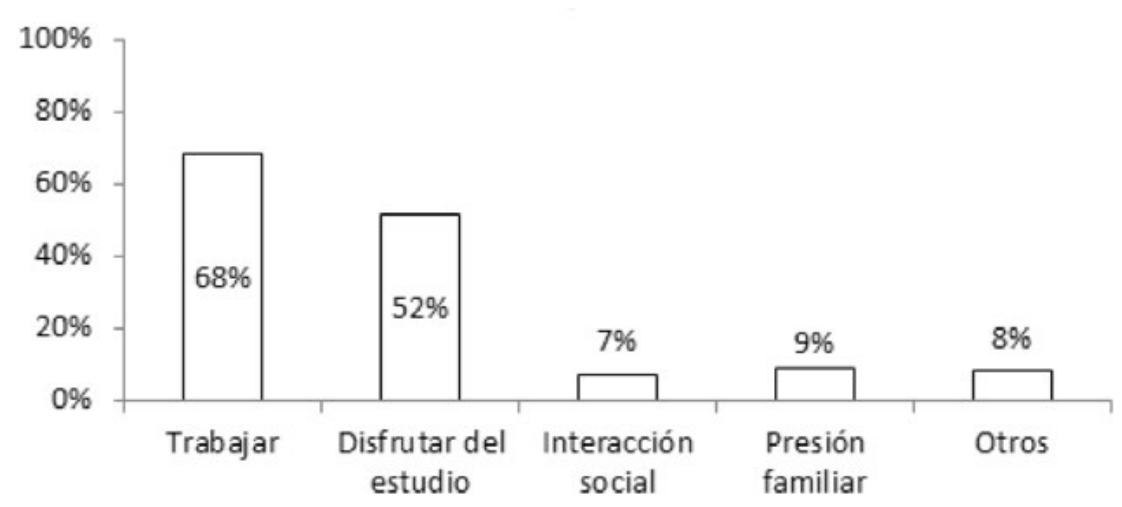

Sobre la base de los motivos expuestos en el párrafo anterior, se realizó la pregunta: ¿Cuáles son tus expectativas para cuando finalices tus estudios? Los resultados se muestran en la Figura 2: Trabajar en lo estudiado $(73 \%, n=131)$ fue la principal expectativa, seguida por Continuar en el deporte $(53 \%, n=95)$ y seguir estudiando $(26 \%, n=47)$. Con puntuaciones menores se encontraron: Trabajar en algo distinto $(4 \%, n=7)$ y Otros $(3 \%, n=5)$.

Asimismo, en la Figura 3 se muestran los resultados de la pregunta: ¿Qué nivel de estudios tienes actualmente?, obteniendo que el $90 \%$ de los deportistas realizan estudios universitarios $(n=161), 7 \%$ estudios técnicos $(n=13)$ y $3 \%$ otra modalidad de estudio.
Figura 2. Expectativas al finalizar los estudios superiores

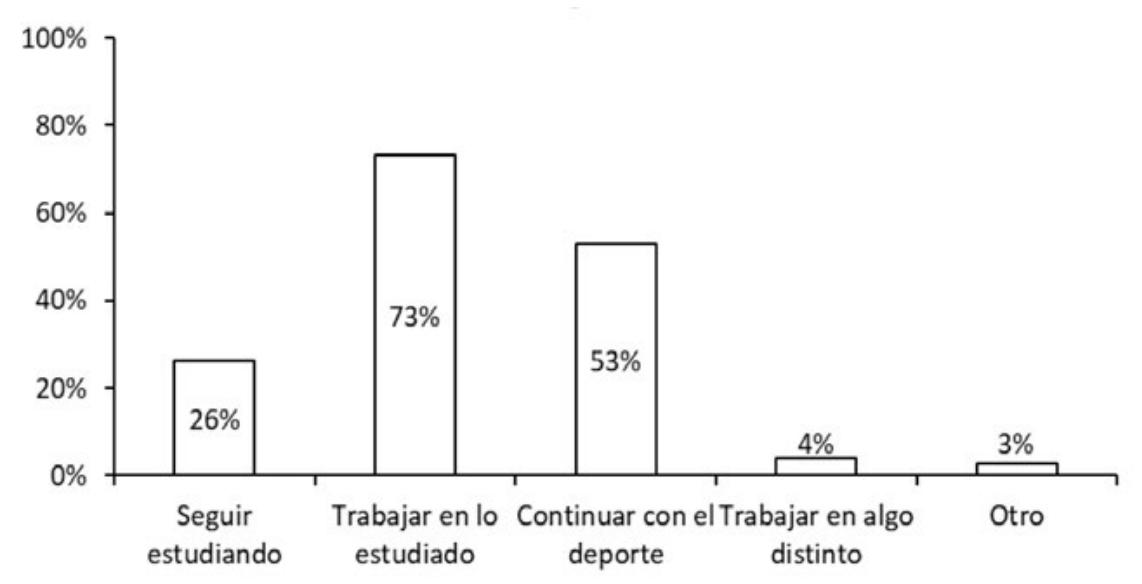

Figura 3. Tipo de estudios superiores que realizan los deportistas

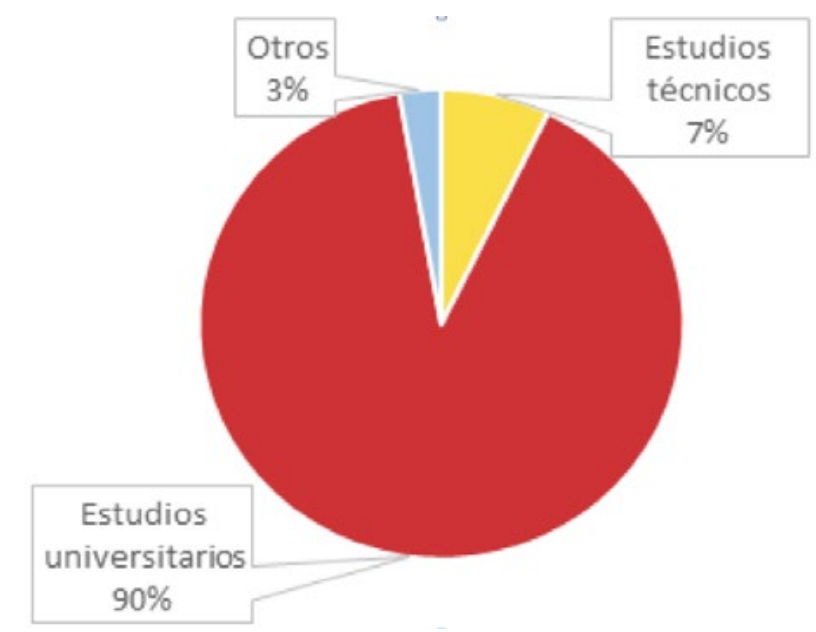

Por otro lado, teniendo en cuenta que el $42 \%$ de los deportistas percibió al factor económico como una dificultad en su carrera deportiva, se recopiló información a partir de la pregunta: ¿Recibes algún tipo de beneficio económico en tu Centro de Estudios? Los resultados se visualizan en la Figura 4 , donde el $42 \%$ de los deportistas no recibió ningún beneficio económico $(n=76), 40 \%$ recibió una beca parcial $(n=71)$, $9 \%$ recibió una beca completa $(n=16), 4 \%$ ayuda económica, $1 \%$ pago de necesidades básicas y $10 \%$ otra modalidad de beneficio.

Figura 4. Beneficios económicos del Centro de Estudios

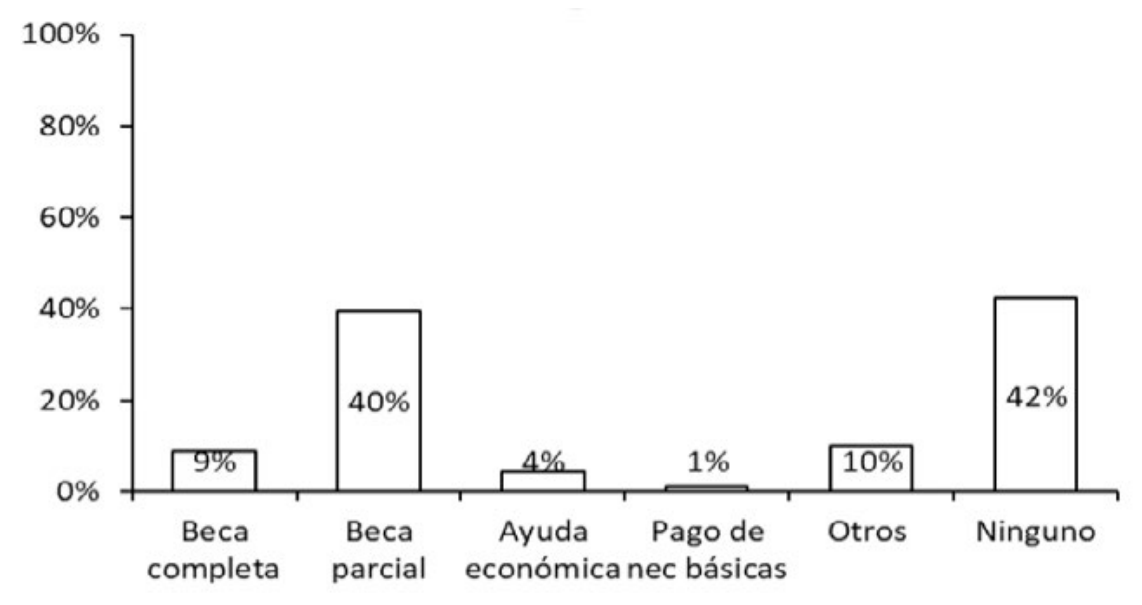

Revista de Psicología Aplicada al Deporte y al Ejercicio Físico (2021), 6, e7, 1-8 


\section{Datos de carrera dual}

De manera complementaria al beneficio económico (con respecto a la carrera académica), otra modalidad de apoyo se da mediante la presencia de un tutor que oriente y asesore al deportista a nivel académico y deportivo, de manera que su papel se torne como un facilitador para compaginar ambas carreras. Es por ello que se preguntó: ¿Cuentas con un tutor deportivo dentro de tu Centro de Estudios? Los resultados encontrados son que el $80 \%(n=143)$ de evaluados no cuenta con tutor deportivo y únicamente el 20\% $(n=36)$ tiene tutor deportivo. (Figura 5)

Figura 5. Deportistas que cuentan con tutor deportivo

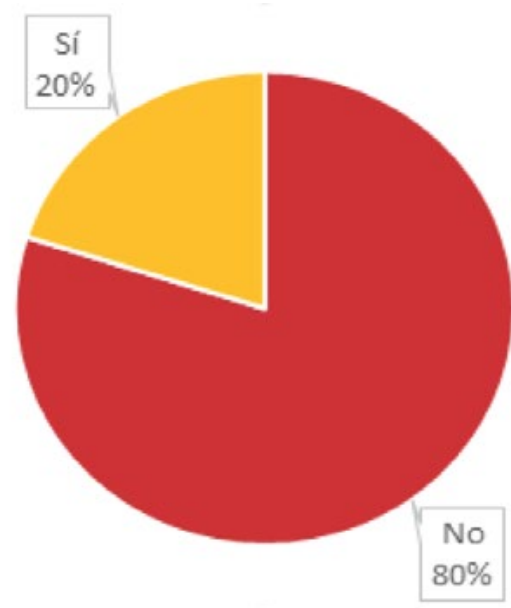

Sin embargo, dentro del ambiente del deportista, el tutor deportivo no es el único agente que influye en la carrera dual, ya que a lo largo de la misma el deportista interactúa con distintas personas. En la Tabla 1 se visualizan los resultados de la pregunta: Enumera en cada uno de los paréntesis del 7 al 6 (Indicando "7" sí es muy importante y " 6 " si es menos importante) el nivel de importancia que atribuyes a cada agente dentro de tu carrera deportiva y académica, encontrándose que la familia es la más importante (54\%), en segundo lugar, los profesores (26\%), seguido por los compañeros y equipo multidisciplinario (25\%), amigos (24\%) y entrenadores (22\%).

Tabla 1. Importancia de los agentes sociales en las carreras duales

\begin{tabular}{cccc}
\hline Puesto & Entorno del deportista & Frecuencia & Porcentaje \\
\hline Puesto 1 & Familia & 96 & $54 \%$ \\
\hline Puesto 2 & Profesores & 47 & $26 \%$ \\
\hline Puesto 3 & Compañeros & 45 & $25 \%$ \\
\hline Puesto 4 & Equipo multidisciplinario & 45 & $25 \%$ \\
\hline Puesto 5 & Amigos & 43 & $24 \%$ \\
\hline Puesto 6 & Entrenadores & 40 & $22 \%$ \\
\hline
\end{tabular}

Así como existen agentes sociales que cumplen el papel de facilitadores, también se presentan barreras u obstáculos que influyen en el desarrollo de la carrera dual. Con respecto a ello, se les preguntó: ¿Qué dificultades experimentas en tu vida deportiva y tus estudios? Encontrándose (Figura 6) que las principales dificultades fueron: el factor Tiempo $(48 \%, n$ = 86) y Económico $(43 \%, n=77)$, seguido del factor Académico $(31 \%, n=56)$, Instalaciones $(26 \%, n=47)$, Personales $(23 \%, n=42)$ y Otros $(11 \%, n=19)$.

Figura 6. Dificultades experimentadas por los deportistas durante su carrera dual

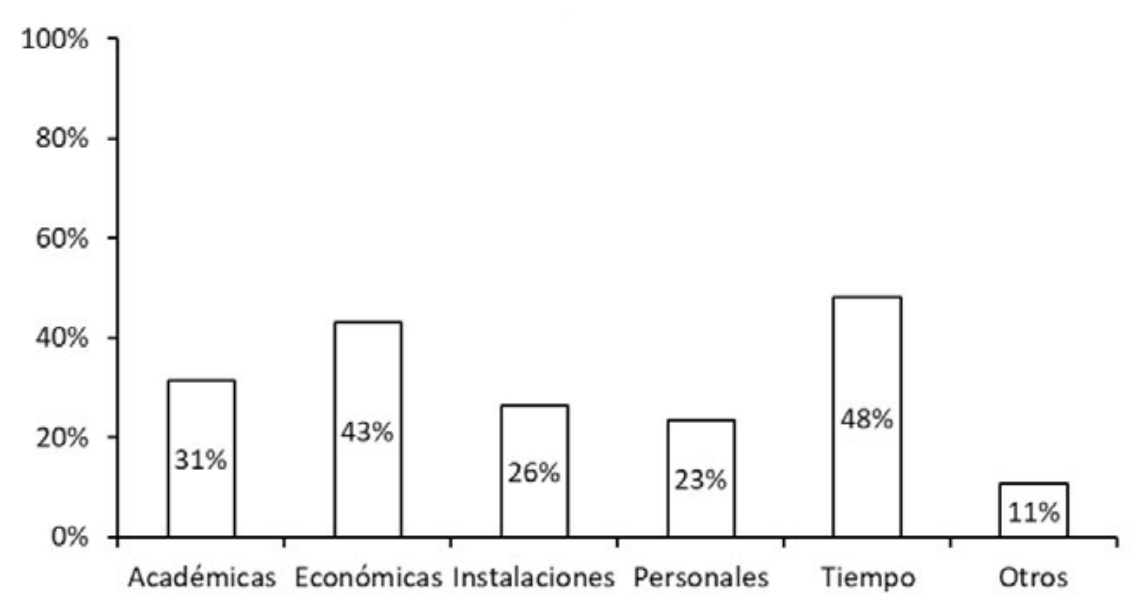

Finalmente, después de realizar la pregunta: ¿Cuál es el nivel de dificultad que tienes o tuviste para llevar tu vida deportiva con tus estudios?, se obtuvo (Figura 7 ) que el $13 \%$ de los deportistas $(n=24)$ percibe como Muy difícil la carrera dual, $43 \%(n=77)$ la cataloga como Difícil, 37\% $(n=66)$ como Regular, 4\% ( $n=8)$ como Fácil y 2\% $(n=4)$ como Muy fácil.

Figura 7. Percepción del grado de dificultad de la carrera dual

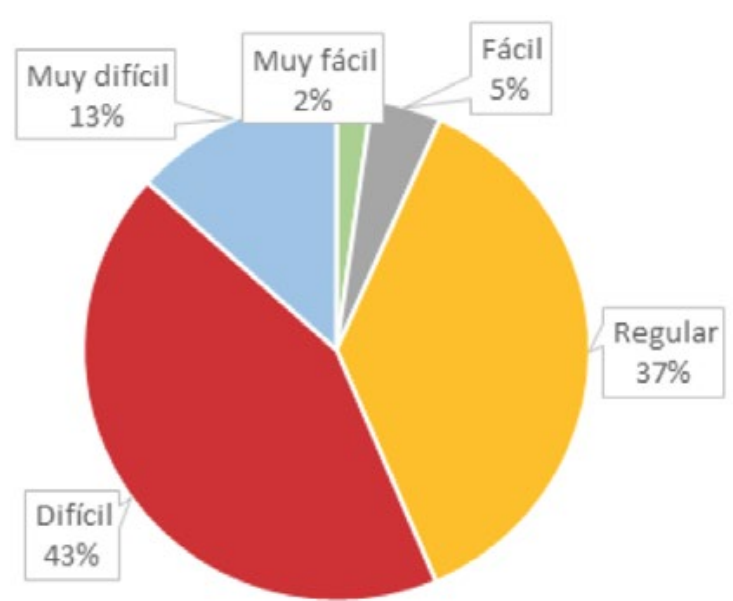




\section{Discusión}

Los deportistas del presente estudio, con referencia a los modelos de trayectoria deportiva descrito por Pallarés et al. (2011), siguen un modelo paralelo, ya que buscan mantener el equilibrio entre la carrera deportiva y académica $(90 \%$ de los deportistas de la investigación realizan estudios universitarios), siendo ambas de prioridad para el deportista. Asimismo, realizar un modelo paralelo requiere diversos esfuerzos en la gestión del tiempo, presentándose situaciones en donde finalizar los estudios se ve condicionado al calendario deportivo; además, también exige esfuerzos en la planificación, pues, aunque existe conciencia del proceso de retirada a futuro, los deportistas deben tomar decisiones continuamente ante eventos y/o sucesos que no se consideraron inicialmente. Sin embargo, todos los esfuerzos no se realizan en vano, dado que existe evidencia de que el modelo paralelo favorece una transición fluida (retirada deportiva) hacia contextos laborales alternativos. (Pallarés et al., 2011; Barriopedro et al., 2018).

De los resultados obtenidos, se identificó que el principal motivo para realizar una carrera académica es el trabajar (68\%). Asimismo, las principales expectativas al finalizar los estudios superiores se orientaron a trabajar en lo estudiado (73\%) y a continuar con el deporte (53\%). Al contrastar la información anterior, se observa una relación entre estudiar y poder trabajar en un futuro (carrera académica-laboral). Esto no implica la exclusión u omisión de la carrera deportiva, por el contrario, se continuaría desarrollando una carrera dual, en la que el área laboral reemplazaría a la académica.

De igual manera, resulta relevante el rol que desempeñan las personas afines al entorno del deportista. En el presente estudio, el agente que fue percibido como el más importante por los deportistas fue la familia (56\%), representada por los padres, los cuales según Tessitore et al. (2020) tienden a orientar su apoyo a las necesidades emocionales, estilo de vida, logística y financieras de sus hijos.

Es por ello que la influencia de los padres se orienta como un facilitador, no solo por el apoyo en las distintas áreas, sino que el nivel académico con el que ellos cuentan influye en la toma de decisiones del deportista con respecto a la elección de una carrera académica. Esto es respaldado por Moreno et al. (2020) quienes concluyeron en su investigación que los deportistas cuyos padres tenían estudios superiores, optaban por realizar una carrera dual cursando el tipo de estudios que los padres habían realizado, y que solían elegir carreras no vinculadas directamente al deporte, lo cual configura una identidad multidimensional que facilita la transición en el retiro de la élite deportiva. En el mismo estudio, Moreno et al. (2020) encontraron que los deportistas cuyos padres no contaban con estudios superiores optaban por estudiar carreras regladas o no regladas vinculadas al deporte cuando tomaban la decisión de realizar una carrera dual, estableciéndose una identidad unidimensional exclusiva en el deporte, que dificultaría la transición a la retirada. Lo señalado anteriormente recalca la importancia que representa el papel de los padres durante el desarrollo de la carrera dual, lo cual también influiría indirectamente en el proceso de retirada, ya que el desarrollo de una carrera dual permite percibir de manera más positiva la transición de retirada del deporte que aquellos que únicamente se enfocaron en la realización de una carrera exclusivamente deportiva. (Barriopedro et al. 2018).

La influencia de los padres resulta significativa, Condello et al. (2019) señalan que los principales partidarios en la promoción de las carreras duales son los padres y los entrenadores. Sin embargo, existen múltiples agentes sociales como jugadores, compañeros, profesores, equipo multidisciplinario que también influyen en la construcción de un entorno óptimo de desarrollo y aprendizaje, que favorece la adaptación del deportista a los distintos cambios y que permiten que el proceso de carrera dual sea exitoso (Gledhill y Harwood, 2015).

Por otro lado, en el presente estudio se encontró que el $56 \%$ de los deportistas encuestados calificó como Difícil o Muy difícil la carrera dual, siendo esta percepción del grado de dificultad similar a la que encontraron López de Subijana et al. (2015) donde los deportistas-estudiantes evaluados percibieron la carrera dual como muy difícil (45.7\%) y difícil (44.9\%). La percepción de dificultad de la carrera dual hallada en nuestro estudio, estaría relacionada con la ausencia de becas académico - deportivas o poco apoyo por parte de la institución académica, debido a que se encontró que un $40 \%$ no recibió ningún beneficio económico de la universidad y solo un 37\% recibió becas parciales. Es por ello que el factor económico es percibido como una de las principales dificultades que influyen directamente en la carrera dual (43\%). De manera similar, se encontró que un $80 \%$ de los deportistas del presente estudio no cuentan con un tutor deportivo, lo cual también influiría en el grado de dificultad percibida por ellos. A las becas económicas y la presencia del tutor deportivo, también se suman otras modalidades de apoyo como la flexibilidad a la hora de asistir a clase, además del libre acceso a instalaciones para la práctica deportiva, y la libre elección de créditos académicos, los cuales actúan como facilitadores durante la carrera dual (Blanco y Burillo, 2018). En la presente investigación, las principales dificultades encontradas fueron el tiempo (48\%) y el factor económico (43\%), las cuales están asociadas a situaciones como las largas concentraciones, coincidencia de los entrenamientos con las clases, estrés, 
cansancio, poco tiempo para el ocio, distancia del centro educativo al lugar de entrenamiento y la no compensación económica como deportista (Guirola et al., 2018; Condello et al. , 2019), las cuales aumentarían la complejidad de realizar una adecuada gestión del tiempo entre el deporte, estudios y otros. (López de Subijana et al., 2015).

En consecuencia, la promoción de las carreras duales debe desempeñarse de la mano con la implementación de Programas de Apoyo al deportista, ya que la mayoría de estos perciben el proceso como "Muy difícil" o "Difícil". Al respecto, una alternativa se orienta a la formulación y ejecución de redes nacionales e internacionales que permitan la cooperación y el intercambio de conocimientos y mejores prácticas, de manera que se involucre al Estado, las instituciones deportivas, instituciones académicas, profesionales y agentes sociales de apoyo al deportista durante la carrera dual (Condello et al., 2019). Por lo que, diseñar Programas de Apoyo al deportista en determinados contextos no es suficiente si es que estos no se masifican. En este sentido, Condello et al. (2019) hallaron que la mayoría de los deportistas no habían participado ni estaban familiarizados con los programas de carrera dual.

Con referencia a la implementación y utilidad de los programas, Ryan et al. (2017) en su estudio brindaron a los deportistas algún tipo de apoyo para completar sus estudios y desarrollar su carrera deportiva. Sin embargo, seguían enfrentando dificultades a la hora de desarrollar una carrera dual, como: las largas ausencias fuera del lugar de estudio, las demandas deportivas inflexibles y una aparente falta de comprensión entre el personal docente y los deportistas.

Por lo que, la implementación de un programa debe ser evaluada, de manera que permita verificar su eficacia y pueda replicarse en contextos similares, como lo realizado por Jordana et al. (2019) donde llevaron a cabo un Programa de Asistencia de Carrera Dual (PACD) que integra la práctica aplicada con la evidencia científica mediante la realización de cinco pasos estructurados: a. Actualización documental, b. Análisis de necesidades, c. Intervención, d. Evaluación, y e. Seguimiento, propuestos por Ramis et al. (2019).

\section{Aplicación práctica}

A partir de las ideas expuestas y los resultados obtenidos en la presente investigación, se busca dar a conocer la situación actual de las carreras duales de deportistas-estudiantes en el contexto peruano, de manera que se permita prestar mayor atención a las necesidades de los deportistas y de pie a nuevos estudios e investigaciones vinculadas a esta doble actividad como la diferencia de género, tipos de depor- te o tipo de carreras estudiadas. Así mismo, consideramos que el conocimiento de los datos presentados podría ser utilizados también en la implementación de políticas y programas de desarrollo y acompañamiento a los deportistas y estudiantes por parte de las federaciones, universidades y demás entes deportivos a través de la figura del psicólogo del deporte y de esta manera garantizar el seguimiento y el correcto desempeño deportivo y educativo de los deportistas tanto de manera individual como colectiva.

\section{Referencias}

Álvarez, P.; Hernández, A. y López, D. (2014). Análisis de la compleja relación entre los estudios universitarios y la práctica deportiva de alto rendimiento. Revista de la Educación Superior, 43(169), 69 - 87. https://doi.org/10.1016/j.resu.2014.01.002

Barriopedro, M., López de Subijana, C. y Muniesa C (2018) Insights into life after sport for Spanish Olympians: Gender and career path perspectives. PLOS ONE 13(12) 1-12. https://doi.org/10.1371/journal.pone.0209433

Blanco, P. Burillo, P. (2017). Los deportistas de élite en el sistema universitario español (Elite athletes in the Spanish university system). Retos, 33, 162-168. https://doi.org/10.47197/retos. v0i33.55773

Condello, G., Capranica, L., Doupona, M., Varga, K. y Burk, V. (2019). Dual-career through the elite university student-athletes' lenses: The international FISU-EAS survey. PLOS ONE, 14(10) 1-11. https://doi:10.1371/journal.pone.0223278

Gledhill, A. y Harwood, C. (2015). A holistic perspective on career development in UK female soccer players: A negative case analysis. Psychology of Sport and Exercise, 21, 65-77. https://doi:10.1016/i.psychsport.2015.04.003

Gómez, M. (2015). Introducción a la metodología de la investigación científica (2da edición). Brujas.

Guirola, I., Torregrosa, M., Ramis, Y. y Jaenes, J. C. (2016). Remando contracorriente: facilitadores y barreras para compaginar el deporte y los estudios. Revista Andaluza de Medicina del Deporte, 11(1) 12-17. https://doi.org/10.1016/j.ramd.2016.08.002

Jordana, A., Pons, J., Borrueco, M., Pallarés, S. y Torregrossa, M. (2019). Implementación de un Programa de Asistencia de Carrera Dual en un Club multideportivo privado. Revista de Psicología Aplicada al Deporte y el Ejercicio Físico, 4(2), Artículo e9, 1-7. https://doi.org/10.5093/rpadef2019a8

López de Subijana, C., Barriopedro, M. y Conde, E. (2015). Supporting dual career in Spain: Elite athletes' barriers to study. Psychology of Sport and Exercise, 21, 57-64. https://doi.org/10.1016/j. psychsport.2015.04.012

Moreno, R., Chamorro, J. y López de Subijana, C. (2020) «Nunca pensé abandonar los estudios»: Relación entre la formación académica de los progenitores y la carrera dual de deportistas de élite. Revista de Psicología del Deporte, 29(2) 17-26.

Moreno, R., Chamorro, J. y López de Subijana, C. (2018) Carrera dual en deportistas de alto nivel españoles: La importancia del apoyo social familiar en el ámbito académico. Revista Española de Educación Física y Deportes, 421, 83-99. 
Pallarés, S., Azócar, F., Torregrosa, M., Selva, C. y Ramis, Y. (2011). Modelos de trayectoria deportiva en waterpolo y su implicación en la transición hacia una carrera profesional alternativa. Cultura, Ciencia y Deporte, 6(17), 93-103. https://doi.org/10.12800/ ccd. v6i77.36

Ramis, Y., Torregrossa, M., Pallarés, S., Viladrich, C. y Cruz, J. (2019). El modelo GEPE de práctica basada en la evidencia: integrando la evidencia científica en la práctica aplicada. Revista de Psicología Aplicada al Deporte y el Ejercicio Físico, 4(2), Artículo e13. https://doi.org/10.5093/rpadef2019a12

Ryan, C., Thorpe, H., y Pope, C. (2017). The policy and practice of implementing a student-athlete support network: a case study. International Journal of Sport Policy and Politics, 9(3), 415-430. https://doi:10.1080/19406940.2017.1320301

Sánchez-Pato, A., Calderón, A., Arias-Estero, J. L., García-Roca, J. A., Bada, J., Meroño, L., Isidori, E., Brunton, J., Decelis, A., Koustelios, A., Mallia, O., Fazio, A., Radcliffe, J. y Sedgwick, M. (2016). Diseño y validación del cuestionario de percepción de los estudiantes universitarios-deportistas de alto nivel sobre la carrera dual (ESTPORT). Cultura, Ciencia y Deporte, 17(32), 127-147.
Sánchez-Pato, A., Conde Pascual, E., Meroño García, L., Arias Estero, J. L. y García-Roca, J. A. (2018). Estudio del éxito académico de un modelo universitario de carrera dual en deportistas-estudiantes según género, nivel de estudios y deporte. Revista Española de Educación Física y Deportes, 421, 35-47.

Stambulova, N., Engström, C., Franck, A., Linnér, L. y Lindahl, K. (2015) Searching for an optimal balance: Dual career experiences of Swedish adolescent athletes, Psychology of Sport and Exercise, 21, 4-14. https://doi.org/10.1016/j.psychsport.2014.08.009

Tessitore, A., Capranica, L., Pesce, De Bois, N., Gjaka, M., Warrington, G., MacDonncha, C., Doupona, M. (2020) Parents About Parenting Dual Career Athletes: A Systematic Lite- rature Review. Psychology of Sport and Exercise, 53(4), Artículo 101833. https://doi:10.1016/j.psychsport.2020.101833

Torregrosa, M., Chamorro, J., y Ramis, Y. (2016) Transición de junior a senior y promoción de carreras duales en el deporte: una revisión interpretativa. Revista de Psicología Aplicada al Deporte y al Ejercicio Físico, 1(1), Artículo e6. https://doi.org/10.5093/rpadef2016a6

Wylleman, P. (2019). An organizational perspective on applied sport psychology in elite sport. Psychology of Sport and Exercise, 42, 89-99. https://doi.org/10.1016/j.psychsport.2019.01.008 